\title{
Laccases stabilization with phosphatidylcholine liposomes
}

\author{
Meritxell Martí ${ }^{*}$, Andrea Zille $^{2}$, Artur Cavaco-Paulo $^{3}$, José Luís Parra $^{1}$, Luisa Coderch ${ }^{1}$ \\ ${ }^{1}$ Institute of Advanced Chemistry of Catalonia (IQAC-CSIC), Barcelona, Spain; \\ *Corresponding Author: meritxell.marti@iqac.csic.es \\ ${ }^{2}$ IBMC-Institute for Molecular and Cell Biology, Porto, Portugal \\ ${ }^{3}$ Textile Engineering Department, University of Minho, Guimarães, Portugal
}

Received 13 October 2011; revised 24 November 2011; accepted 8 December 2011

\begin{abstract}
In recent years, there has been an upsurge of interest in enzyme treatment of textile fibres. Enzymes are globular proteins whose catalytic function is due to their three dimensional structure. For this reason, stability strategies make use of compounds that avoid dismantling or distorting protein 3D structures. This study is concerned with the use of microencapsulation techniques to optimize enzyme stabilization. Laccases were embedded in phophatidylcholine liposomes and their encapsulation capacity was assessed. Their enzymatic activity and stability were analyzed, comparing free-enzymes, enzymes in liposomes, and the lipid fraction separated from the aqueous fraction. An increase in their encapsulation efficiency was found at higher lipid/laccase ratios. Relative activity of enzyme-containing vesicles has also been shown to be retained much more than that of free native enzymes. The loss of activity of laccases entrapped in the vesicles in the total stability process is lower than $10 \%$ compared with $40 \%$ to $60 \%$ of loss of free-laccases after heating the samples for 3 days. Laccase stabilization could be of interest to future textile or cosmetic applications because of their potential for environmentally friendly oxidation technologies.
\end{abstract}

Keywords: MLV Liposome; Enzymes; Laccases; Encapsulation; Stability

\section{INTRODUCTION}

Enzymes have been used in different industries including textiles for washing, scouring, dyeing, etc. Protein enzymes can interact with all products used in the process, and their large 3D structure enables their interaction with different chemical products in solution due to the variety of side chains of the amino acids. Enzyme stabilization has assumed considerable importance owing to the increasing number of enzyme applications and to the need for realizing their full potential as catalysts [1].

Liposomes are defined as a structure composed of lipid vesicle bilayers enclosing an aqueous volume. These structures have long been used as carrier systems for the delivery of vaccines, therapeutic drugs and hormones because of easy preparation, good biocompatibility, low toxicity and commercial availability [2,3]. Efficient functioning of enzymes inside liposomes opens up new possibilities of applications in biocatalysis and bioanalytical tools [4-6]. It has been observed that enzymes are considerably stabilized within the nano-environment of liposomes since they are protected from unfolding and proteolysis. Liposomes can effectively protect enzymes from aggression of external agents such as proteases [7]. In addition, enzymes entrapped in liposomes are stabilized against unfolding forces owing to hydrophobic interactions between the enzyme and the liposome membrane [8]. Moreover, enzymes encapsulated inside liposomes retain their activity even at very low concentrations [9]. Since liposomes are optically translucent, they can be used as optical sensor elements [10]. Novel liposome-based nano-sized biosensor systems have been prepared by encapsulating an enzyme using porin embedded in the lipid membrane. As a result, the enzyme activity within the liposome can be monitored using pyranine as a fluorescent $\mathrm{pH}$ indicator [11]. Furthermore, some enzymes such us horseradish peroxidase encapsulated in liposomes have been directly detected without lysis using Luminol chemiluminiscence [12].

There are two main areas of application of enzymecontaining lipid vesicles: biomedicine (enzyme-replacement therapy) and the food industry (cheese ripening process). In both cases the lipid vesicles are carriers that protect the enzymes from contact with blood or milk, respectively [6]. The stability effect of enzyme encapsu- 
lation in liposomes has been studied for many enzymes in these two fields [6]. However, few studies have been performed with laccases that are used in textile and cosmetic industries [13-15].

Laccases are of special interest because of their ability to oxidise both phenolic and non-phenolic lignin related compounds as well as other environmental pollutants, which makes them very useful for biotechnologies. The use of laccases in textiles is currently enjoying rapid growth; they are used for decolorizing textile effluents [16,17], bleaching [18], dyeing [19], synthesizing dyes [20] and for modifying the surface of fabrics [21,22]. In the cosmetic field, laccases can replace $\mathrm{H}_{2} \mathrm{O}_{2}$ as an oxidizing agent in dye formulation [23]. Enzymes are globular proteins whose catalytic function is due to their three-dimensional conformation. For this reason, stability strategies make use of compounds that avoid dismantling or distorting protein 3D structures. Therefore, microencapsulation, particularly with liposomes, can be envisaged as a good strategy for stabilizing laccases. Of the different existing oxidant enzymes, laccases have been the subject of intensive research in the last decades due to their low substrate specificity in the textile and cosmetic fields as stated above. Therefore, new strategies are needed for stabilizing and maintaining their enzymatic activity.

Liposomes have also aroused a great deal of interest in the textile industry $[24,25]$ and in the cosmetic industry $[26,27]$. This work seeks to shed light on the behaviour of laccases microencapsulated in liposomes. Enzyme stabilization was determined by the evaluation of the thermostability of the free and entrapped laccases. Efficient functioning of laccases inside liposomes would open up new avenues for textile or cosmetic applications.

\section{MATERIALS AND METHODS}

\subsection{Liposomes Formation and Enzime Encapsulation}

Liposome suspension of $2 \mathrm{wt} \%$ of phosphatidylcholine (PC) and $10 \mathrm{wt} \%$ laccases solution (weight ratio of lipid to laccase $1 / 5$ lipid/laccases (LpLc)) and also another suspension of $10 \mathrm{wt} \%$ of PC and $5 \mathrm{wt} \%$ of laccases solution (weight ratio of lipid to laccase 1/0.5 lipid/laccases (LpLc)) were prepared using the film hydration method. To obtain these liposome suspensions, $0.1 \mathrm{~g}$ or $0.5 \mathrm{~g}$, respectively of Lipoid S-100 (Lipoid GmbH, Germany) were prepared in solution in $30 \mathrm{ml}$ of chloroform. The chloroform was then removed by a rotary evaporator under reduced pressure. A thin film of lipid was observed after the solvent were removed. The lipid film was hydrated with $5 \mathrm{ml}$ buffer solution (pH 5) containing the laccase solution, $5.86 \mathrm{mg} / \mathrm{ml}$, commercial Trametes villosa of Novozymes Spain S.A., $(0.5 \mathrm{ml}$ or $0.25 \mathrm{ml}$, respectively) and, after 10 minutes sonication in a water bath multilamellar vesicles (MLV) were obtained. Liposomes suspensions of $2 \mathrm{wt} \%$ and $10 \mathrm{wt} \%$ of PC (Lp) without enzymes were also prepared following the same methodology but using only a buffer solution without laccases.

The amount of protein was determined for the laccase (Lc) solution and also for the lipid alone to evaluate possible interferences. To quantify the laccases entrapped in liposomes, LpLc was precipitated and separated from the supernatant by centrifugation at 14000 RPM for 15 minutes using a Centrifuge 5415-Eppendorf (Germany). After separation, the supernatant was retained. The precipitate was filled at $1 \mathrm{ml}$ with buffer and agitated vigorously and centrifuged again. This process was repeated two more times and all supernatants were kept together ( $\mathrm{Sn}$ LpLc). Finally, the eppendorf with the precipitate liposomes (V LpLc), the supernatant (Sn LpLc) and the full LpLc solution, were filled with a $0.125 \%$ Triton X-100 solution, (octylphenol ethoxylated with 10 units of ethylene oxide and active matter of $100 \%$ ) supplied by Tenneco S.A. (Spain), and agitated vigorously to solubilise the lipid bilayer. The amount of protein was determined in all samples following the Bradford method in order to obtain the amount of encapsulated laccase. The formulations evaluated are summarized in Figure 1.

The Bradford method was used to quantify the protein in these samples. It is based on the formation of a complex between the dye, Brilliant Blue G, Sigma (USA), and the proteins in solution, which produces an increase

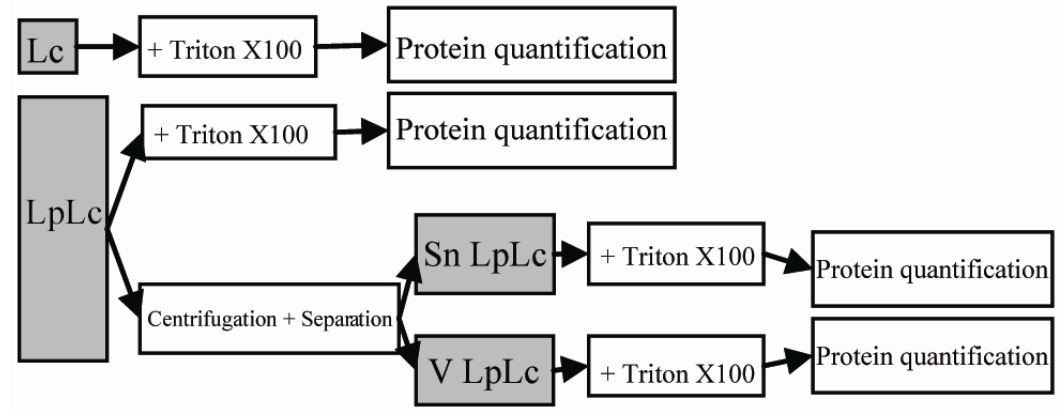

Figure 1. Diagram of the protein quantification and of stability assays. 
in absorption at $595 \mathrm{~nm}$ and is proportional to the protein present $[28,29]$. To calculate the amount of protein, BSA (Bovine Serum Albumin) from Sigma (USA) was used as a standard.

\subsection{Enzime Activity and Stability Assay}

Enzyme activity (U) is defined as $\mu$ mol of substrate oxidized per min. Activity assay was performed using ABTS. The assay mixture contains 0.0005M 2,2'-azinobis (3-ethylbenzthiazoline-6-sulfonate) (ABTS) provided by Sigma (USA), $0.1 \mathrm{M}$ sodium acetate buffer $\mathrm{pH} 5$, and a suitable amount of enzyme or liposome-enzyme. Laccase activity was assayed spectrophotometrically by measuring the increase in absorbance at $420 \mathrm{~nm}\left(\varepsilon_{420}=\right.$ $3.6 \times 10^{4} \mathrm{M}^{-1} \cdot \mathrm{cm}^{-1}$ ) owing to the oxidation of ABTS [30]. When laccases were encapsulated in liposomes, the liposomes were solubilised with $10 \%$ Triton X-100. In all cases $0.1 \mathrm{~mL}$ of sample which has lipids (LpLc, Sn LpLc and V LpLc) was solubilised with $0.3 \mathrm{~mL}$ of $10 \%$ Triton $\mathrm{X}-100$ before the spectrophotometric assay. The stability assessment was made by performing the activity assay for three days following the graph in Figure 1. To increase the experimental thermal conditions, the different samples were heated at $60^{\circ} \mathrm{C}$ for 120 minutes between each activity assay. The results were obtained from triplicate assays.

\section{RESULTS AND DISCUSSION}

\subsection{Liposome Formation and Enzime Encapsulation}

There are a number of methods that can be used for the preparation of enzyme-containing lipid vesicles (liposomes) that are lipid dispersions that contain watersoluble enzymes in the trapped aqueous space. A review of these studies indicates that the most widely used vesicle-forming amphiphiles are based on phosphatidylcholine [6]. Moreover, encapsulation of enzymes in lipid vesicles has been performed by using a variety of different vesicle preparation methods [6]. The dispersion of a dry lipid film in an enzyme-containing aqueous solution leads to the formation of a relative polydisperse vesicle suspension with mainly large, multilamellar vesicles (MLV). The preparation is not very reproducible because the resulting size distribution and lamellarity very much depends on the quality of the lipid film and on the way this film is dispersed. Since most of these vesicles are multilamellar, water-soluble enzymes can be localized not only in the central core but also in the aqueous interlamellar spaces, resulting in relatively high encapsulation efficiency. A number of studies on enzyme-containing MLV have been carried out, e.g. with many enzymes, but to our knowledge, not with Laccases. Therefore, lipo- somes of $2 \%$ of phosphatidylcholine (PC) and $10 \%$ laccases (1/5 lipid/laccases (LpLc)) and also 10\% of PC and $5 \%$ of laccases (1/0.5 lipid/laccases (LpLc)) were prepared using the dry lipid film hydration method described in the experimental part. Multilamellar vesicles were obtained.

The Bradford method was used to determine the amount of enzymes in the vesicles and in the supernatant solution of the original liposomes with laccases (LpLc) for use in the enzyme activity assay. Accordingly, the following formulations were prepared: the two liposomes with laccases (LpLc), (1/5 and 1/0.5 lipid laccases) containing $10 \%$ and $5 \%$ of laccase solution, respectively; the two laccases only in buffer solution (Lc) containing 10 and $5 \%$ of laccase, respectively; and the two liposomes in buffer solution, without enzymes, (Lp) with 2 and $10 \%$ PC in order to determine possible interferences of the lipid compound. LpLc was centrifuged to separate the precipitated vesicles from the supernatant. The protein content of all the solutions was evaluated. Significant interferences were found between the phosphatidylcholine of the LpLc solutions and the Bradford reagent. This interaction was more marked with the latter formulation (LpLc 1/0.5, with 10\% of PC) because of the high proportion of lipids with the result that the findings were less reliable.

The results for the formulation with $2 \%$ of lipid and $10 \%$ of laccases LpLc (1/5) showed that the enzyme encapsulated accounted for $8 \%$. When the proportion of lipid increases LpLc (1/0.5) the enzyme encapsulation reaches almost 15\% (Table $\mathbf{1}$ ).

Enzyme entrapment is in general directly proportional to lipid concentration $[6,31,32]$. In our case, even only

Table 1. Laccase formulations, enzyme encapsulation, enzymatic activity $(\mathrm{U} / \mathrm{mL})$ and enzymatic activity percentages after a thermal process.

\begin{tabular}{|c|c|c|c|c|c|c|}
\hline Form & dation & & & & $1 / 0$ & $\mathrm{Lc}$ \\
\hline \% Cor & apounds & & Pс $2 \%$ & c $10 \%$ & Pc $10^{\circ}$ & c 5\% \\
\hline Encap & sulation & & & & & \\
\hline & & & $\mathrm{U} / \mathrm{ml}$ & $\%$ & $\mathrm{U} / \mathrm{ml}$ & $\%$ \\
\hline & & $1^{\mathrm{st}}$ & 50.0 & 100 & 25.0 & 100 \\
\hline & Lc & $2^{\text {nd }}$ & 35.1 & 70.2 & 19.2 & 76.8 \\
\hline & & $3^{\text {rd }}$ & 20.5 & 41.0 & 14.4 & 57.6 \\
\hline & & $1^{\mathrm{st}}$ & 44.18 & 100 & 22.2 & 100 \\
\hline & LpLc & $2^{\text {nd }}$ & 36.654 & 82.99 & 18.8 & 84.4 \\
\hline $\begin{array}{c}\text { Enzymatic } \\
\text { Activity }\end{array}$ & & $3^{\text {rd }}$ & 24.471 & 55.38 & 17.2 & 77.5 \\
\hline & & $1^{\mathrm{st}}$ & 23.64 & 100 & 12.155 & 100 \\
\hline & Sn LpLc & $2^{\text {nd }}$ & 22.271 & 94.21 & 10.43 & 84 \\
\hline & & $3^{\text {rd }}$ & 19.722 & 83.43 & 8.271 & 67.8 \\
\hline & & $1^{\mathrm{st}}$ & 5.113 & 100 & 6.648 & 100 \\
\hline & V LpLc & $2^{\text {nd }}$ & 5.36 & 100 & 6.781 & 100 \\
\hline & & $3^{\text {rd }}$ & 4.98 & 97.4 & 5.95 & 89.5 \\
\hline
\end{tabular}


two lipid/laccases ratios were studied, but a clear increase on encapsulation percentage with the increase on lipid concentration was obtained. The encapsulation efficiency of protein has been reported to depend on interaction between the protein and the lipid bilayer [32]. The enzyme entrapment can be increased by manipulation of the liposomal lipid composition or by increasing the lipid concentration, in order to favour electrostatic interactions [32]. Sometimes the entrapment efficiency decreases with increasing the lipid content [31]. The addition of more lipid increases the lamelarity of the vesicle population rather than producing more vesicles of the same lamelarity [31]. Therefore, modification of lipid composition and lipid/enzyme ratios would be the base of further work with the aim to improve encapsulation efficiency and to deep inside the protein-lipid interactions.

Similar results have been reported for enzyme entrapment efficiency of other enzymes in MLV. These varied in most cases from below 5\% [33-35] to about 15\% [36]. Thus, although a smaller amount of enzymes and a lower activity could be found in the liposome formulation of $10 \%$ of lipid and $5 \%$ of laccases LpLc (1/0.5), the higher encapsulation obtained could demonstrate more clearly the effect of the liposome on the stability of the encapsulated laccases.

\subsection{Enzime Activity and Stability Assay}

Laccase activity was evaluated by the ABTS method as detailed in the experimental part. The same amount of laccases in liposomes and laccases in the buffer solution of $5 \%$ and $10 \%$ were assessed. As expected, the enzyme activity of the solution containing $10 \%$ of laccases $(50.0$ $\mathrm{U} / \mathrm{ml}, 10 \% \mathrm{Lc}$ ) is approximately double that of the solution containing $5 \%$ laccases $(24.9 \mathrm{U} / \mathrm{ml}, 5 \% \mathrm{Lc}$ ) since the enzyme concentration is also the double. In both cases, the presence of liposomes leads to a decrease in the enzyme activity of about $11.6 \%$ for $1 / 5 \mathrm{LpLc}(44.2 \mathrm{U} / \mathrm{ml}$, 10\% LpLc) and $11.2 \%$ for 1/0.5 LpLc (22.2 U/ml, 5\% LpLc). These decreases in laccase activities when formulated with PC liposomes were expected because the barrier of the lipid membrane diminished the activity of the enzyme entrapped in the liposomes [37]. It should be borne in mind that formulations with a high amount of enzymes are more prone to lose activity in the thermal or proteolytical processes. Therefore, the similar percentage of lost activity found for the two formulations could be due to the high PC content in the 1/0.5 LpLc (10\%Pc) and to the high amount of Laccases in 1/5 LpLc formulation (10\% Laccases).

Even though enzyme activity is decreased in the initial LpLc formulation when lipids were present, the stabilities of the two formulations were assayed. The main aim of this work was to study a possible increase in enzyme stability in its formulation entrapped in liposomes. The influence of enzyme entrapment on enzyme stability was also investigated. To assess stability, a protocol was designed to compare laccase stability in buffer solution (Lc) and laccase stability formulated with liposomes (LpLc) (Figure 1). In addition to evaluating the total formulation of laccases in liposomes, aliquots of the formulation were centrifuged to separate the supernatant (Sn LpLc) from the pellet (V LpLc) (in which laccases are encapsulated) and the activities of the two samples were also determined. To toughen the conditions, samples were heated for 2 hours at $60^{\circ} \mathrm{C}$ and the activity was measured the following day. This process was continued for three successive days.

The enzyme activity graph of the different samples obtained during the three days of the experiment is shown in Figure 2. The first day of the experiment during which the samples were not heated, the laccases in the liposome solution presented a lower activity than the laccases in buffer solution, as discussed above. However, after 3 days, the LpLc formulation maintained higher activity values than the free laccases Lc. The graph clearly shows the stabilization obtained when laccases are bound to liposome vesicles. Relative activity of other enzymecontaining vesicles has also been demonstrated to retain activity much longer than native enzyme [31] as in our case. The stabilization of the supernatant should also to be noted. However, higher stabilization was obtained for the enzyme entrapped in the vesicles. The two formulations show similar activity $(5-7 \mathrm{U} / \mathrm{ml})$ for the Laccases entrapped in the vesicles. This occurs because the enzyme entrapment is double in this formulation despite the presence of only $50 \%$ of the Laccase concentration in the 1/0.5 LpLc.

The influence of the liposomes on the stability of laccase activity is clearly demonstrated by the percentage of laccase activity of each sample during the whole stability process (Table 1). The greater stabilization of the laccases that are exclusively entrapped in the vesicles should be noted. The activity loss of laccases entrapped in the vesicles in the total stability process is lower than $10 \%$ when compared with $40 \%$ to $60 \%$ of activity of free-laccases after heating the samples for 3 days. These results confirm that the increase in the stability undergone by laccases in the liposome solution is mainly due to the encapsulation degree of the enzymes.

In general, encapsulation of enzymes in liposomes has demonstrated the enhancement of their stability versus denaturizing [6,9]. For example, other enzymes such as amylogucosidase entrapped in multilamellar vesicles (MLVs) composed of other phospholipids such as dipalmitoylphosphatidylcholine (PPC) have been much more stable than free enzymes [38]. The rate of hydroly- 


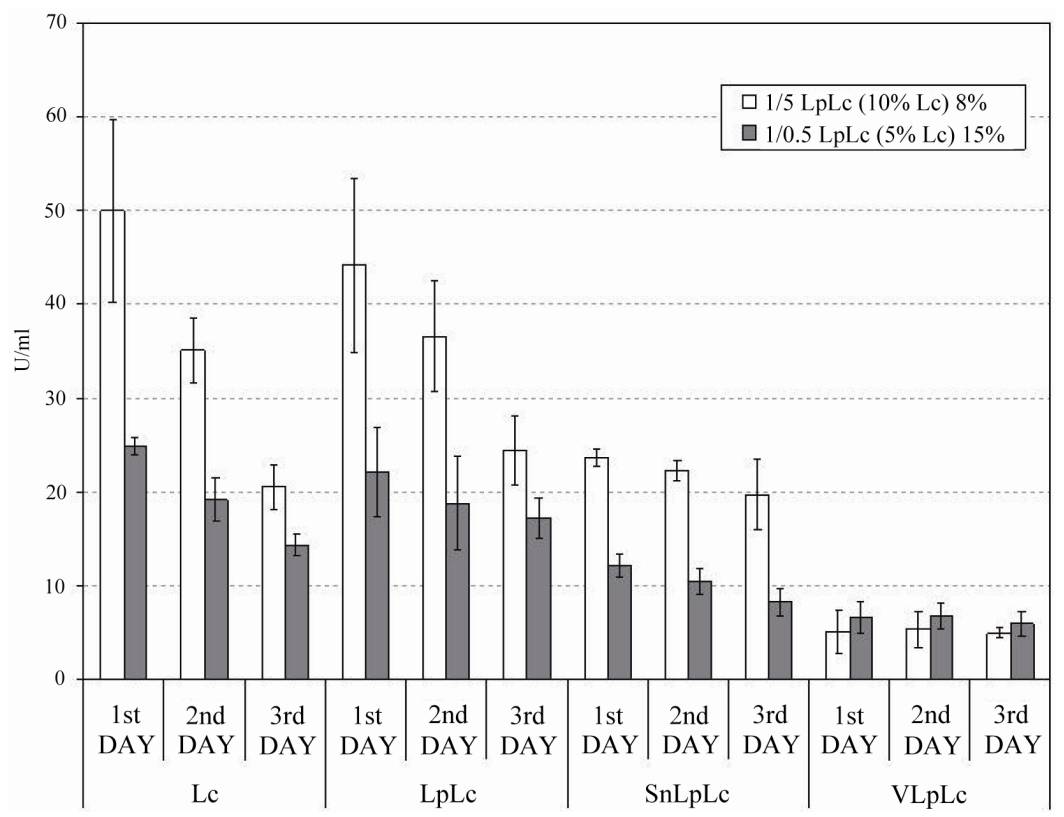

Figure 2. Enzyme activity (U/ml) and their error bars (standard deviation) of laccases in buffer solution (Lc), laccases in liposome formulation (LpLc), supernatant of LpLc (Sn LpLc) and vesicles of LpLc (V LpLc), of 1/5 LpLc and 1/0.5 LpLc during the stability assay.

sis is relatively low because of the low permeability of substrate across the liposome bilayer [31]. Moreover, glucose oxidase encapsulated with phosphatidylcholine and cholesterol liposomes has shown that the thermal and proteolytic stabilities are also enhanced by encapsulation in liposomes [38]. Some studies indicate that the interaction of the enzymes with the vesicle membrane is based on the lipid vesicle assistance the refolding of unfolded enzymes [319 of (6)].

However, the activity of enzyme entrapped in the liposome is markedly reduced by the permeability barrier of the lipid membrane, resulting in a lower internal concentration of the substrate. In order to overcome this problem, some authors have investigated the permeabilization of the wall of the liposome [9].

Currently there are generally two approaches to increase the substrate permeability. One is to reconstitute membrane channel proteins in the liposome bilayers while the other is to utilize lipid/detergent hybrid membranes (27 of 31). Exploiting the protective ability of lipid nanocontainers in combination with controlled permeability by modified channels, could open new future applications.

In our work, we present the stabilization behaviour of another enzyme, the laccase whose use in textile and in cosmetics, as stated in the Introduction, is growing rapidly $[18,20,22,23,39]$. Stabilization and its relation with the encapsulation degree are demonstrated. Development of an effective system for laccase encapsulation has lately deserved great attention to retain activity [40-43].
However, none of those works used vesicles formed with phospholipids to encapsulate laccases. Further studies will focus on modifying the lipid composition and/or lipid/enzyme proportion to increase enzyme encapsulation, diminish enzyme permeation with the aim of enhancing enzyme stabilization.

\section{CONCLUSIONS}

Laccases were microencapsulated in phosphatidylcholine MLV liposomes in different lipid/enzyme proportions. Their encapsulation efficiency was evaluated, indicating $8 \%$ of encapsulation when $1 / 5$ lipid/laccases were assayed versus $15 \%$ of encapsulation with $1 / 0.5$ lipid/laccases. This demonstrates the increase in encapsulation efficiency at higher lipid/laccases ratios.

Enzyme activity showed a decrease of about $11 \%$ for the two formulations, 1/5 lipid/laccases and 1/0.5 lipid/ laccases with respect to the laccases in buffer solution. This decrease in laccase activity when formulated with PC liposomes was expected given the barrier effect of the lipid bilayers of the vesicles.

However, evaluation of the activity loss of laccases entrapped in the vesicles during the thermal process demonstrated an increase in stability undergone by laccases in the liposome solution. This increase is much more marked in the case of the laccases encapsulated in the vesicles free of supernatant, which confirms the effect of the liposome encapsulation on the continued activity of the enzyme. The use of phospholipidic lipo- 
somes in the laccases encapsulation warrants further studies that modify the lipid composition and/or lipid/ enzyme proportion to increase enzyme encapsulation and consequently enzyme stabilization. The study of other lipid/laccases ratios would allow us to determine their possible linearity with encapsulation efficiency and with enzyme activity as it happen with other enzymes and phospholipids [31,32]. Laccase stabilization could be of considerable interest to future textile or cosmetic applications owing to their potential for environmentally friendly oxidation technologies.

\section{ACKNOWLEDGEMENTS}

The authors are indebted to Ms. I. Yuste for technical support. The authors also wish to thank G. von Knorring for improving the final version of the manuscript. This work was supported by FCT (SFRH/ BPD/37045/2007; PTDC/CTM/100627/2008), QREN, COMPETEPrograma Operacional Factores de Competitividade na sua componente FEDER.

\section{REFERENCES}

[1] Iyer, P.V. and Ananthanarayan, L. (2008) Enzyme stability and stabilization-aqueous and non-aqueous environment. Process Biochemistry, 43, 1019-103.

doi:10.1016/j.procbio.2008.06.004

[2] Gregoriadis, G., Florence, A.T. and Patel, H.M. (1993) Liposomes in drug delivery. Harwood Academic Publishers, London.

[3] Gregoriadis, G. (1999) DNA vaccines: A role for liposomes. Current Opinion in Molecular Therapeutics, 1, 39-42.

[4] Rosenberg, M.F., Jones, M.N. and Vadgama, P.M. (1991) A liposomal enzyme electrode for measuring glucose. Biochimica et Biophysica Acta, 1115, 157-165.

[5] Walde, P. and Marzetta, B. (1998) Bilayer permeability-based substrate selectivity of an enzyme in liposomes. Biotechnology and Bioengeering, 57, 216-219. doi:10.1002/(SICI)1097-0290(19980120)57:2<216::AIDBIT10>3.3.CO;2-2

[6] Walde, P. and Ichikawa, S. (2001) Enzymes inside lipid vesicles: Preparation, reactivity and applications, Biomololecular Engineering, 18, 143-177. doi:10.1016/S1389-0344(01)00088-0

[7] Winterhalter, M., Hilty, C., Bezrukov, S.M., Nardin, C., et al. (2001) Controlling membrane permeability with bacterial porins: Application to encapsulated enzymes. Talanta, 55, 965-971. doi:10.1016/S0039-9140(01)00494-5

[8] Han, X., Li, G. and Li, K. (1998) FTIR study of the thermal denaturation of a-actinin in its lipid-free and dioleoylphosphatidylglycerol-bound states and the central and $\mathrm{N}$-terminal domains of a-actinin in $\mathrm{D}_{2} \mathrm{O}$. Biochemistry, 37, 10730-10737. doi:10.1021/bi9800451

[9] Nasseau, M., Boublik, Y., Meier, W., Winterhalter, M., et al. (2001) Substrate-permeable encapsulation of enzymes maintains effective activity, stabilizes against denaturation and protects against proteolytic degradation. Biothechnology and Bioengeering, 75, 615-618. doi:10.1002/bit.10074

[10] Kulin, S., Kishore, R., Helmerson, K. and Locascio, L. (2003) Optical manipulation and fusion of liposomes as microreactors. Langmuir, 19, 8206-8210. doi:10.1021/la0344433

[11] Vamvakaki, V., Fournier, D. and Chaniotakis, N.A. (2005) Fluorescence detection of enzymatic activity within a liposome based nanobiosensor. Biosensors and Bioelectronics, 21, 384-388. doi:10.1016/j.bios.2004.10.028

[12] Kamidate, T., Komatsu, K., Tani, H. and Ishida, A. (2008) Direct determination of horseradish peroxidase encapsulated in liposomes by using luminol chemiluminescence. Analytical Sciences, 24, 477-481. doi:10.2116/analsci.24.477

[13] Minussi, R.C., Pastore, G.M. and Durán, N. (2002) Potential applications of laccase in the food industry. Trends in Food Science \& Technology, 13, 205-216. doi:10.1016/S0924-2244(02)00155-3

[14] Kuhad, R.C., Singh, A. and Eriksson, K.E.L. (1997) Advances in biochemical engineering biotechnology. In: Eriksson, K.E.L., Ed., Biotechnology in the Pulp and Paper Industry, Springer Verlag, Berlin.

[15] Rodríguez Couto, S. and Toca Herrera, J.L. (2006) Industrial and biotechnological applications of laccases: A review. Biotechnology Advances, 24, 500-513. doi:10.1016/j.biotechadv.2006.04.003

[16] Zille, A., Tzanov, T., Gübitz, G. and Cavaco-Paulo, A. (2003) Immobilized laccase for decolourization of reactive black 5 dyeing. Biotechnology Letters, 25, 14731477. doi:10.1023/A:1025032323517

[17] Zille, A., Górnacka, B., Rehorek, A. and Cavaco-Paulo, A. (2005) Degradation of azo dyes by trametes villosa laccase over long periods of oxidative conditions. Applied and Environmental Microbiology, 71, 6711-6718. doi:10.1128/AEM.71.11.6711-6718.2005

[18] Vinoid, S. (2001) Enzymatic decolourisation of denims: A novel approach. Colourage, 48, 25-26.

[19] Tzanov, T., Silva, C.J., Zille, A., Oliveira, J. and CavacoPaulo, A. (2003) Effect of some process parameters in enzymatic dyeing of wool. Applied Biochemistry and Biothecnology, 111, 1-13. doi:10.1385/ABAB:111:1:1

[20] Setti, L., Giuliani, S., Spinozzi, G. and Pifferi, P.G. (1999) Laccase catalyzed-oxidative coupling of 3-methyl 2-benzothiazolinone hydrazone and methoxyphenols. Enzyme and Microbial Technology, 25, 285-289. doi:10.1016/S0141-0229(99)00059-9

[21] Silva, C., Silva, C.J., Zille, A., Güebitz, G.M. and CavacoPaulo, A. (2007) Laccase immobilization on enzymatically functionalized polyamide 6,6 fibres. Enzyme and Microbial Technology, 41, 867-875. doi:10.1016/j.enzmictec.2007.07.010

[22] Kim, S.Y., Zille, A., Murkovic, M., Güebitz, G. and Cavaco-Paulo, A. (2007) Enzymatic polymerization on the surface of the functionalized cellulose fibres. Enzyme and Microbial Technology, 40, 1782-1787. 
doi:10.1016/j.enzmictec.2007.01.001

[23] Roure, M., Delattre, P. and Froger, H. (1992) Composition for an enzymatic coloration of keratin fibres, especially for hair and its use in a dyeing process. European Patent Application EP0504005.

[24] Montazer, M., Validi, M. and Toliyat, T. (2006) Influence of temperature on stability of multilamellar liposomes in wool dyeing. Journal of Liposome Research, 16, 81-89. doi:10.1080/08982100500528883

[25] Martí, M., de la Maza, A., Parra, J.L. and Coderch, L. (2001) Dyeing wool at low temperatures: New method using liposomes. Textile Research Journal, 71, 678-682. doi:10.1177/004051750107100805

[26] De Pera, M., Coderch, L., Fonollosa, J., De la Maza, A., et al. (2000) Effect of internal wool lipid liposomes on skin repair. Skin pharmacol. Applied Skin Physiology, 13, 188- 195. doi:10.1159/000029925

[27] Ramírez, R., Martí, M., Cavaco-Paulo, A., Siva, R., De la Maza, A., Parra, J.L. and Coderch, L. (2009) Liposome formation with wool lipid extracts rich in ceramides. Journal of Liposome Research, 19, 77-83. doi:10.1080/08982100802538838

[28] Bradford, M.M. (1976) A rapid and sensitive method for the quantization of microgram quantities of protein utilizing the principle of protein-dye binding. Analytical Biochemistry, 72, 248-254. doi:10.1016/0003-2697(76)90527-3

[29] Technical Bulletin of Bradford Reagent, Sigma-Aldrich (USA).

[30] Childs, R.E. and Bardsley, W.G. (1975) The steady-state kinetics of peroxidase with 2, 2'-azino-di-(3-ethyl-benzthiazoline-6-sulphonic acid) as chromogen. Biochemical Journal, 145, 93-103.

[31] Li, M., Handford, M.J., Kim, J.W. and Peeples, T.L. (2007) Amyloglucosidase enzymatic reactivity inside lipid vesicles. Journal of Biological Engineering, 1, 4. doi:10.1186/1754-1611-1-4

[32] Colletier, J-P., Chaize, B., Winterhalter, M. and Fournier, D. (2002) Protein encapsulation in liposomes: Efficiency depends on interactions between protein and phospholipid bilayer. BMC Biotechnology, 2, 9. doi:10.1186/1472-6750-2-9

[33] Kirby, C.J., Brooker, B.E. and Law, B.A. (1987) Accelerated ripening of cheese using liposome-encapsulated enzymes. International Journal of Food Science and Technology, 22, 355-375. doi:10.1111/j.1365-2621.1987.tb00499.x

[34] Naoi, M., Naoi, M., Shimizu, T., Malviya, A. and Yagi, K. (1977) Permeability of amino acids into liposomes. Biochimica et Biophysica Acta, 471, 305-310. doi:10.1016/0005-2736(77)90258-9

[35] Law, B.A. and King, J.S. (1985) Use of liposomes for Proteinase addition to Cheddar cheese. Journal of Dairy Research, 52, 183-188. doi:10.1017/S0022029900024006

[36] Alkhalaf, W., Piard, J-C., El Soda, M., Gripon, J-C., Desmazeaud, M. and Vassal, L. (1988) Liposomes as proteinase carriers for the accelerated ripening of saintpaulin type cheese. Journal Food Science, 53, 1674-1679. doi:10.1111/j.1365-2621.1988.tb07813.x

[37] Chaize, B., Colletier, J.P., Winterhalter, M. and Fournier, D. (2004) Encapsulation of enzymes in liposomes. High encapsulation efficiency and control of substrate permeability. Artificial cell blood subtitutes and biomed. Biotechnology, 32, 67-75.

[38] Rodriguez-Nogales, J.M. (2004) Kinetic Behavior and stability of glucose oxidase entrapped in liposomes. Journal of Chemical Technology and Biotechnology, 79, 7278. doi:10.1002/jctb.944

[39] Kunamneni, A., Ghazi, I., Camarero, S., Ballesteros, A. and Plou, F.J. (2008) Decolorization of synthetic dyes by laccase immobilized on epoxy-activated carriers. Process Biochemistry, 43, 169-178. doi:10.1016/j.procbio.2007.11.009

[40] Khani, Z., Jolivalt, C., Cretin, M., Tingry, S. and Innocent, C. (2006) Alginate/carbon composite beads for laccasse and glucose oxidase encapsulation: Application in biofuel cell technology. Biotechnology Letters, 28, 1779-1786. doi:10.1007/s10529-006-9160-1

[41] Lloret, L., Eibes, G., Feijo, G., Moreira, M.T., Lema, J.M. and Hollman, F. (2011) Immobilization of laccase by encapsulation in a sol-gel matrix and its chrarcterization and use for the removal of estrogens. Biotechnology Progress, 27, 1570-1579. doi:10.1002/btpr.694

[42] Niu, J., Yin, L. and Jiang, F. (2011) In situ encapsulation of laccase in nanofibers by electrospinning for development of enzyme biosensors for chlorophenol monitoring. Analyst, 136, 4802-4808.

[43] Mazur, M., Krywko-Cendrowska, A., Krysinski, P. and Rogalski, J. (2009) Encapsulation of laccase in a conducting polymer matrix: A simple route towards polypyrrole microcontainers. Synthetic Metals, 159, 1731-1738. doi:10.1016/j.synthmet.2009.05.018 